\section{The Incidence of Arterial and Venous Thrombosis in Antineutrophil Cytoplasmic Antibody-associated Vasculitis}

\section{To the Editor:}

We read with interest in The Journal of Rheumatology the article by Kang, et al, "High Incidence of Arterial and Venous Thrombosis in Antineutrophil Cytoplasmic Antibody-associated Vasculitis," which reported a dramatically high incidence of arterial (ATE) and venous thrombosis events (VTE) in a hospital-based cohort of patients with antineutrophil cytoplasmic antibody-associated vasculitis (AAV). The study generally supports the previously reported increased risk of ATE and VTE in patients affected by $\mathrm{AAV}^{2-8}$, but has a number of inaccuracies and methodological issues that inflate the incidence rates of ATE and VTE.

First, the authors compared clinical and laboratory features at baseline between groups defined by events that occurred during the followup (ATE vs no ATE, and VTE vs no VTE, Table 1 and 2 , respectively) ${ }^{1}$, and used Kaplan-Meier curves to compare the overall survival between groups defined by these time-dependent variables. Because ATE and VTE occurred during the followup after a diagnosis of AAV was established, they should not be used to stratify the patient cohort at baseline; a patient could change groups if an event occurred during a longer followup.

Second, although it is well documented that a history of previous ATE predicts the risk of subsequent ATE in both the general population and $\mathrm{AAV}^{5,9}$, this study ${ }^{1}$ did not exclude patients with prior ischemic events from the risk analyses, thus very likely leading to overestimation of the risk of ATE in patients with AAV. Accordingly, the sensitivity analysis that the authors performed after excluding patients with strokes and ischemic heart disease events prior to AAV diagnosis showed indeed a lower incidence, albeit still elevated, of ATE compared to the original analyses [1.26 instead of 1.56/100 person-yrs (PY) for coronary events, and 1.06 instead of $1.10 / 100 \mathrm{PY}$ for ischemic stroke].

Third, the authors compared the rates of ATE and VTE in AAV to the crude rates in the general UK population, without adjustment to ensure comparability. Event rates based on different age, sex, and calendar year distributions will not be comparable. AAV overall and particularly microscopic polyangiitis and renal-limited vasculitis (up to one-third in this study population) usually affect adults and older populations, thus potentially affecting these comparisons.

The lingering question is why ATE and VTE might be so much more common in this cohort. In addition to the methodological issues, the authors failed to adequately provide a sound biological explanation for their findings regarding this particular aspect of AAV epidemiology. An examination of known risk factors would be helpful, but as acknowledged in the discussion, there were no data on lipid profiles, statin treatment, and body mass index at baseline.

We previously showed that although cardiovascular risk factors were similarly distributed between patients with AAV and matched comparators at baseline (indeed, cholesterol blood levels might be even lower in patients, resembling the "lipid paradox" described for rheumatoid arthritis), they were strongly associated with the subsequent risk of developing ATE in patients with $\mathrm{AAV}^{2}$. While unusual in other reports of AAV, the possibility of a concomitant diagnosis of antiphospholipid syndrome as the cause of thromboembolic and cardiovascular complications was not ruled out ${ }^{10}$. Given the extremely high ATE and VTE rates reported by Kang and colleagues ${ }^{1}$, these factors might have been relevant to explain the results they obtained.

Finally, the authors claimed that their article was the first to report a higher incidence of ischemic stroke in AAV. However, in May 2018 we had already published a paper demonstrating that the risk for ischemic cerebrovascular accidents was 8-fold higher in patients with incident AAV than matched comparator subjects (HR 8.16,95\% CI 2.45-27.15)2

In this study ${ }^{1}$ the incidence estimates for ATE and VTE may be inflated and the validity of its conclusion jeopardized. Accurate and useful estimates rely on correct application of epidemiological methodology and consideration of relevant biological factors.
ALVISE BERTI , MD, Division of Pulmonary and Critical Care Medicine, Mayo Clinic College of Medicine and Science, Rochester, Minnesota, USA, and Santa Chiara Hospital, Trento, CIBIO, University of Trento, Trento, Italy; ERIC L. MATTESON, MD, MPH, Division of Rheumatology, and Division of Epidemiology, Department of Health Sciences Research, Mayo Clinic College of Medicine and Science; CYNTHIA S. CROWSON ${ }^{\circ}$, PhD, Division of Biomedical Statistics and Informatics, Department of Health Sciences Research, and Division of Rheumatology, Mayo Clinic College of Medicine and Science; ULRICH SPECKS, MD, Division of Pulmonary and Critical Care Medicine, Mayo Clinic College of Medicine and Science; DIVI CORNEC, MD, PhD, Division of Pulmonary and Critical Care Medicine, Mayo Clinic College of Medicine and Science, Rochester, Minnesota, USA, and INSERM UMR1227, Lymphocytes B et Autoimmunité, Université de Bretagne Occidentale, CHU de Brest, Brest, France. Address correspondence to Dr. D. Cornec, CHU Brest, Rheumatology, Boulevard Tanguy Prigent, Brest, 29609, France.E-mail: divi.cornec@chu-brest.fr

\section{REFERENCES}

1. Kang A, Antonelou M, Wong NL, Tanna A, Arulkumaran N, Tam FW, et al. High incidence of arterial and venous thrombosis in antineutrophil cytoplasmic antibody-associated vasculitis. J Rheumatol 2019;46:285-93.

2. Berti A, Matteson EL, Crowson CS, Specks U, Cornec D. Risk of cardiovascular disease and venous thromboembolism among patients with incident ANCA-associated vasculitis: a 20-year population-based cohort study. Mayo Clin Proc 2018;93:597-606.

3. Avina-Zubieta JA, Mai A, Amiri N, Dehghan N, Ann Tan J, Sayre $\mathrm{EC}$, et al. Risk of myocardial infarction and stroke in patients with granulomatosis with polyangiitis (Wegener's): a population-based study. Arthritis Rheumatol 2016;68:2752-9.

4. Faurschou M, Ahlstrom MG, Lindhardsen J, Baslund B, Obel N. Impact of pre-existing co-morbidities on mortality in granulomatosis with polyangiitis: a cohort study. Rheumatology 2016;55:649-53.

5. Morgan MD, Turnbull J, Selamet U, Kaur-Hayer M, Nightingale P, Ferro CJ, et al. Increased incidence of cardiovascular events in patients with antineutrophil cytoplasmic antibody-associated vasculitides: a matched-pair cohort study. Arthritis Rheum 2009;60:3493-500.

6. Suppiah R, Judge A, Batra R, Flossmann O, Harper L, Hoglund P, et al. A model to predict cardiovascular events in patients with newly diagnosed Wegener's granulomatosis and microscopic polyangiitis. Arthritis Care Res 2011;63:588-96.

7. Merkel PA, Lo GH, Holbrook JT, Tibbs AK, Allen NB, Davis JC Jr, et al; Wegener's Granulomatosis Etanercept Trial Research Group. Brief communication: high incidence of venous thrombotic events among patients with Wegener granulomatosis: the Wegener's Clinical Occurrence of Thrombosis (WeCLOT) Study. Ann Intern Med 2005;142:620-6.

8. Mourguet M, Chauveau D, Faguer S, Ruidavets JB, Béjot Y, Ribes $\mathrm{D}$, et al. Increased ischemic stroke, acute coronary artery disease and mortality in patients with granulomatosis with polyangiitis and microscopic polyangiitis. J Autoimmun 2019;96:134-41.

9. George MG, Tong X, Bowman BA. Prevalence of cardiovascular risk factors and strokes in younger adults. JAMA Neurol 2017;74:695-703

10. Sebastian JK, Voetsch B, Stone JH, Romay-Penabad Z, Lo GH, Allen NB, et al; Wegener's Granulomatosis Etanercept Trial Research Group. The frequency of anticardiolipin antibodies and genetic mutations associated with hypercoagulability among patients with Wegener's granulomatosis with and without history of a thrombotic event. J Rheumatol 2007;34:2446-50.

J Rheumatol First Release July 1 2019; 2019;46:9; doi:10.3899/jrheum.181351 